Aline Sarturi Ponte ${ }^{1}$ Elenir Fedosse ${ }^{1}$
${ }^{1}$ Programa de PósGraduação em Distúrbios da Comunicação Humana, Centro de Ciências da Saúde, Universidade Federal de Santa Maria. Secretaria do PPGDCH Prédio 26/1418/4 andar, Cidade Universitária. 97105-900 Santa Maria RS Brasil. alinesarturi@hotmail.com

\section{Lesão Encefálica Adquirida: impacto na atividade laboral de sujeitos em idade produtiva e de seus familiares}

\author{
Acquired Brain Injury: impacts on labor activity \\ of individuals of working age and their relatives
}

Abstract This study correlated the impact of Acquired Brain Injury (ABI) on the labor activity of individuals of working age on their quality of life (QOL) and that of their relatives/caregivers. It involved qualitative (content analysis) and quantitative (descriptive) research. Forty-eight individuals with $A B I$ and 27 relatives/caregivers were interviewed. The correlation of the International Classification of Functioning, Disability and Health (ICF) and the World Health Organization Quality of Life WHOQOL-BREF (52.1\% men; mean age 48.7 years at the time of the injury; low schooling; $18,7 \%$ domestic) revealed that the Mental Functions compromised by ABI interfered in the four domains of QOL; Heart Functions and Blood Pressure compromised the social domain; Cerebral injury interfered with the Psychological and Environmental domains. With respect to relatives/caregivers $(77.7 \%$ women; 96.3\%, primary caregivers; mean age 52.6 years, and low schooling), we observed changes in the work routine and family structure (85.2\%). The onset of ABI disrupts the injured individuals and their families, affecting QOL in all domains, especially in the physical, psychological and environmental domains of individuals with $A B I$ and the physical and environmental domains of relatives/ caregivers.

Key words Quality of Life, Caregivers, Functioning, Disability and Health
Resumo Este estudo correlacionou o impacto da Lesão Encefálica Adquirida (LEA) na atividade laboral de sujeitos em idade produtiva à sua Qualidade de Vida (QV) e de seus familiares/ cuidadores. Pesquisa quali-quantitativa. Para a análise dos dados qualitativos realizou-se Análise de Conteúdo e os quantitativos foram analisados descritivamente. Foram entrevistados 48 sujeitos com LEA e 27 familiares/cuidadores. A correlação da Classificação Internacional de Funcionalidade, Incapacidade e Saúde - WHOQOL-BREF (52,1\% homens; média de idade 48,7 anos, no momento da lesão; nível escolar baixo; 18,7\% doméstica) revelou que as Funções Mentais comprometidas pela LEA interferiram nos quatro domínios da QV; Funções do Coração e Pressão Sanguínea acometeram o dominio Social; a lesão Cerebral interferiu nos domínios Psicológico e Ambiental. Quanto aos familiares/cuidadores (77,7\% mulheres; 96,3\% informais; média de idade 52,6 anos; e nível escolar baixo), observaram-se alterações na rotina de trabalho e na estrutura familiar (85,2\%). O surgimento de uma LEA desorganiza o sujeito lesionado e sua família, afetando a $Q V$ em todos os seus dominios, especialmente nos físicos, psicológicos e ambientais do primeiro e os domínios físicos e ambientais de familiares/cuidadores.

Palavras-chave Qualidade de vida, Cuidadores, Funcionalidade, Incapacidade e saúde 


\section{Introdução}

Há claras indicações de que o nível de independência e autonomia de uma pessoa reflete na sua condição de uso linguístico/comunicativo, nas Atividades de Vida Diária (AVD), Atividades Instrumentais de Vida Diária (AIVD) e nas atividades de lazer e de trabalho. Uma Lesão Encefálica Adquirida (LEA) pode causar dificuldades de comunicação ou ruptura de interações sociais, e aumento da dependência nas atividades cotidianas ${ }^{1}$.

Sendo assim, no período imediato à sobrevivência a uma LEA, o sujeito acometido pode apresentar deficiências físicas, mentais e/ou sensoriais, tais são definidas Organização Mundial da Saúde - OMS como, "problemas nas funções ou nas estruturas do corpo como um desvio significativo ou uma perda" ${ }^{2}$. Neste sentido, as diferentes condições de vida associadas às deficiências adquiridas após a lesão tendem a alterar o potencial do sujeito para manter um senso positivo de bem-estar subjetivo ${ }^{3}$.

Pode-se dizer que, devido aos aspectos anteriormente mencionados, os lesionados encefálicos deparam-se com uma importante diminuição da Qualidade de Vida $(\mathrm{QV})^{1}$. De acordo com a OMS, a QV é definida como "a percepção do indivíduo de sua posição na vida, no contexto da cultura e sistema de valores nos quais ele vive e em relação aos seus objetivos, expectativas, padrões e preocupações" ${ }^{\text {. }}$. Deste modo, pode-se compreender o termo QV como: a satisfação do sujeito em realizar suas atividades cotidianas, em manter seus relacionamentos sociais, como a família e amigos, e também, ter acesso à educação, à saúde, ao trabalho, ao lazer e condições salubres de moradia e trabalho ${ }^{5,6}$.

As sequelas decorrentes de uma LEA exigem do sujeito grande esforço adaptativo - enfrentamento de desafios, provenientes de um evento não desejado, que causam desequilíbrio no funcionamento físico, social, psicológico e ambien$\mathrm{tal}^{7}$. O sucesso na adaptação às demandas impostas por uma LEA é um importante indicador do estado de bem-estar e do senso de ajustamento pessoal.

Autores como Panhoca e Gonçalves ${ }^{8}$ esclarecem que as pessoas acometidas por LEA são afetadas não apenas pelas incapacidades que limitam suas atividades básicas, mas também pela dificuldade que encontram para voltar ao trabalho, o que determina forte declínio no bem-estar global, dada a importância que o trabalho tem, não só para a sobrevivência, como também para o autoconceito, o status social e as relações sociais.
Este estudo ocupou-se das sequelas da LEA nas estruturas e funções corporais, nas atividades básicas cotidianas e atividades trabalhistas, analisando o impacto de tais sequelas nos domínios da QV de sujeitos acometidos pela LEA e de seus familiares. Em outras palavras, este estudo correlacionou o impacto da LEA na atividade laboral de sujeitos em idade produtiva a suas QV, bem como à de seus familiares.

\section{Métodos}

Este estudo é de natureza quali-quantitativa. Foi aprovado pelo Comitê de Ética em Pesquisa em Seres Humanos, da Universidade onde se realizou a pesquisa.

Participaram deste estudo 48 sujeitos com LEA e 27 familiares/cuidadores. O período de coleta de dados foi entre junho e dezembro de 2014. Participaram deste estudo sujeitos em idade produtiva (16 a 60 anos de idade para mulheres e 16 a 65 anos de idade para homens ${ }^{9}$ ), de ambos os sexos, com LEA (afásicos e/ou com sequelas motoras), inseridos em serviços de reabilitação de Fisioterapia, Fonoaudiologia e/ou Terapia Ocupacional que integram o sistema público (serviços municipais, federais, privados e filantrópicos que prestam atendimentos pelo Sistema Único de Saúde) de uma cidade da região central do Rio Grande do Sul. Também participaram familiares/ cuidadores, de ambos os sexos (acima de 18 anos de idade). Os sujeitos com LEA foram identificados como S1, S2, S3 etc. e seus familiares/cuidadores F1, F2, F3 etc., preservando-se, assim, a identidade dos participantes.

O primeiro contato da pesquisadora com os sujeitos e seus familiares/cuidadores foi realizado por intermédio de um terapeuta da clínica responsável pelo atendimento, ocasião em que se realizava o convite para participarem da pesquisa e se marcava um encontro, no domicílio dos sujeitos, quando, então, estes e seus familiares/cuidadores eram convidados a assinar o Termo de Consentimento Livre e Esclarecido (TCLE) e a responderem a um questionário semiestruturado (elaborado especialmente para esta pesquisa), à Classificação Internacional de Funcionalidade, Incapacidade e Saúde (CIF) e ao World Health Organization Quality of Life -Bref (WHOQOL - Bref). Os familiares/cuidadores, após consentirem com a pesquisa, respondiam a um questionário semiestruturado (também elaborado para fins desta pesquisa) e ao WHOQOL - Bref. Contou-se com o auxílio de um gravador Sony Icd-Px240 para a coleta de dados. 
O questionário semiestruturado aplicado aos sujeitos com LEA continha questões relacionadas ao sexo, idade na época da lesão, idade atual, diagnóstico, sequelas, escolaridade, categoria de segurado, situação diante da previdência social, profissão, carga horária de trabalho, tempo de afastamento, se foi realizada alguma tentativa de retorno ao trabalho, tempo de afastamento, se permaneceu na mesma função ou foi remanejado de cargo, orientações para o retorno ao trabalho, adaptações no local de trabalho, dificuldade no retorno ao trabalho, barreiras atitudinais, alterações no cotidiano após a lesão. A entrevista semiestruturada realizada com os familiares/cuidadores dos sujeitos com LEA foi composta pelos seguintes questionamentos: sexo, idade, profissão, escolaridade, parentesco, cuidador principal, alterações na estrutura familiar e no cotidiano.

A CIF está dividida em duas partes, subdivididas em dois componentes cada. A primeira aborda os aspectos da Funcionalidade e Incapacidade - Funções e Estruturas do Corpo, e a segunda destaca os Fatores Contextuais - Fatores Ambientais e Pessoais. Devido ao fato de o foco da CIF ser nos aspectos funcionais, esta avaliação (por assim dizer) adquire um caráter multidisciplinar, o que permite ser aplicada em todas as culturas, valorizando assim o contexto no qual o sujeito está inserido ${ }^{10}$.

O WHOQOL - Bref avalia a qualidade de vida pela percepção do próprio sujeito, considerando seus aspectos subjetivos e seu contexto cultural e social. É composto por 26 itens distribuídos em quatro domínios - i) físico; ii) psicológico; iii) relações sociais; e, iv) meio ambiente ${ }^{11}$.

Para a análise dos dados qualitativos dos sujeitos com LEA e de seus familiares, utilizou-se a Análise de Conteúdo - um conjunto de técnicas de investigação que, através de uma descrição objetiva, sistemática e quantitativa do conteúdo manifesto nas comunicações, tem por finalidade a interpretação destas ${ }^{12}$. Para a realização de tal análise, os dados foram primeiramente transcritos, criando-se unidade/tópicos de registros; após, foram criadas categorias.

No caso dos sujeitos com LEA, foram criadas duas categorias: reinserção laboral e condições atuais de vida cotidiana (alterações na atividade de trabalho, sentimentos em relação ao afastamento, alterações nas AVD e AIVD, limitações nas atividades de lazer). No caso dos familiares/ cuidadores foram criadas três categorias: mudança na estrutura familiar (inversão de papéis no circulo familiar, aceitação das mudanças pela família, afastamento e/ou aproximação de familia- res), sobrecarga - física, psicológica e emocional - decorrente da existência de um membro com LEA na família e percepção do familiar/cuidador sobre o afastamento da atividade laboral do sujeito com LEA e seus impactos sobre a vida do sujeito e dos familiares.

A interpretação dos dados, explorou cada categoria.

Os dados quantitativos foram analisados pelo Software Statistica 9.1, da seguinte forma: i) Teste de Correlação de Spearman, correlacionando-se cada variável da CIF com cada domínio do WHOQOL - Bref; dos sujeitos com LEA; ii) Teste Não Paramétrico U de Mann-Whitney para comparar os resultados do WHOQOL - Bref dos sujeitos com LEA com os de seus familiares/ cuidadores e iii) análise descritiva de frequência dos dados dos questionários semiestruturados. Todos os testes estatísticos realizados neste estudo consideraram o nível de significância de 5\% $(\mathrm{p}<0,05)$.

\section{Resultados}

Participaram deste estudo 48 sujeitos com LEA em idade produtiva, sendo $47,9 \%$ do sexo feminino e 52,1\% do sexo masculino; a idade média, no momento da lesão, foi de 48,7 anos.

Quanto à condição civil, identificou-se que 64,5\% eram casados, 22,9\% divorciados, $8,3 \%$ solteiros e $4,3 \%$ viúvos.

O nível escolar foi baixo: 62,5\% tinham o Ensino Fundamental Incompleto, 10,4\% Ensino Fundamental Completo; 2,1\% tinham o Ensino Médio Incompleto; 20,8\% tinham o Ensino Médio Completo; 2,1\% tinham o Ensino Superior Completo; e 2,1\% tinham Pós-Graduação.

Quanto ao diagnóstico, 41,1\% foram acometidos por Acidente Vascular Cerebral isquêmico (AVCi) e 15,3\% por Acidente Vascular Cerebral hemorrágico (AVCh), 37,4\% não souberam informar o tipo de AVC e 6,2\% sofreram Traumatismo Crânio Encefálico (TCE). Dos sujeitos participantes, 52,1\% eram afásicos, 52,2\% apresentavam hemiplegia direita, 41,6\% hemiplegia esquerda, 4,1\% quadriplegia e 2,1\% não apresentavam sequelas motoras.

Considerando que a CIF oportuniza uma grande compilação de dados, optou-se por apresentar, neste estudo, os dados das variáveis correspondentes à Funcionalidade e Incapacidade (Funções e Estruturas do Corpo) e aos Fatores Contextuais (Fatores Ambientais e Pessoais) que apresentaram significância estatística, quando 
correlacionadas com os domínios do WHOQOL - Bref (confira as Tabelas 1 e 2). A Tabela 1 representa as Funções e as Estruturas do Corpo, observando-se as alterações causadas pela LEA que influenciaram significativamente os domínios do WHOQOL - Bref. Constatou-se que as alterações causadas pela LEA nas Funções Mentais interferem em todos dos domínios da qualidade de vida dos sujeitos com lesão neurológica.

O sujeito acometido por uma LEA apresenta, na maioria das vezes, comprometimentos nas funções linguísticos/cognitivas que acarretam limites em suas condições de organizar, planejar e executar as tarefas cotidianas de seu desejo. Estes déficits têm influência direta na condição de comunicação e organização do cotidiano, fragilizando, assim, seus domínios sociais, conforme evidenciado no excerto a seguir, extraído do discurso do S13.

É, memória, é... que mais afetou; eu pego as coisa aqui de... dentro, ferramenta, coisa assim... levo lá pra fora pra fazer... e depois esqueço... Não lembro onde eu guardei ou se eu guardei, simplesmente some da minha memória, some. (S13; em 11/09/2014, no domicílio do sujeito).

As Funções do Sistema Cardiovascular, Hematológico, Imunológico e Respiratório mostraram-se significantes nas Funções do Coração (b410) e na Pressão Sanguínea, fatos que interferem no domínio social (Tabela 1 ).

Quanto às Estruturas do Sistema Nervoso Central, o cérebro (s110), principal estrutura lesada em uma LEA (Tabela 1), apresentou interferência nos domínios psicológico $(p<0,032)$ e ambiental $(\mathrm{p}<0,012)$.

As alterações causadas pela LEA nos Fatores Ambientais (Tabela 2) refletem nas atividades cotidianas dos sujeitos; há comprometimento em todas as áreas da vida prática/cotidiana em decorrência da lesão, a saber: nos Aspectos de Aprendizagem e Aplicação do Conhecimento (d1), nas Tarefas e Demandas Gerais (d2), na Comunicação (d3), na Mobilidade (d4), no Cuidado Pessoal (d5), nas Atividades Domésticas (d6), Interações Interpessoais (d7) e Áreas Principais da Vida (d8).

Nos aspectos de $\mathrm{d} 1$, correlacionados ao WHOQOL - Bref, foram constatadas limitações nos quatro domínios. Algumas atividades, como ler (d166), escrever (d170) e calcular (d172) comprometem de modo importante os domínios da qualidade de vida; o comprometimento $\mathrm{da}$ leitura afetou os domínios psicológicos ( $\mathrm{p}<$ $0,021)$ e sociais $(p<0,002)$; as dificuldades de escrita apresentaram interferência sobre os domínios sociais $(\mathrm{p}<0,002)$ e o ato de calcular afetou

Tabela 1. Os aspectos da Funcionalidade e Incapacidade - Funções e Estruturas do Corpo correlacionados com os Domínios do WHOQOL - Bref $(\mathrm{n}=48)$.

\begin{tabular}{lrc}
\hline \multicolumn{1}{c}{ Variáveis } & Coeficiente (r) & p-valor \\
\hline b1 Funções Mentais & & \\
b1140 Orientação em relação ao tempo X Domínio Físico & $-0,312$ & 0,032 \\
b130 Funções da energia e de impulsos X Domínio Físico & $-0,444$ & 0,002 \\
b130 Funções da energia e de impulsos X Domínio Psicológico & $-0,511$ & 0,002 \\
b130 Funções da energia e de impulsos X Domínio Ambiental & $-0,532$ & 0,002 \\
b134 Sono X Domínio Físico & $-0,412$ & 0,003 \\
b1403 Compartilhar a atenção X Domínio Ambiental & $-0,357$ & 0,012 \\
b144 Memória X Domínio Físico & $-0,386$ & 0,006 \\
b1440 Memória de curto prazo X Domínio Ambiental & $-0,304$ & 0,035 \\
b152 Funções emocionais X Domínio Psicológico & $-0,444$ & 0,002 \\
b152 Funções emocionais X Domínio Social & $-0,302$ & 0,036 \\
b156 Funções da percepção X Domínio Ambiental & $-0,308$ & 0,033 \\
b164 Funções cognitivas superiores X Domínio Ambiental & $-0,357$ & 0,012 \\
b167 Funções mentais da linguagem X Domínio Social & $-0,357$ & 0,012 \\
b4 Funções do Sistema Cardiovascular, Hematológico, Imunológico e Respiratório & & \\
b410 Funções do coração X Domínio Social & $-0,316$ & 0,028 \\
b420 Pressão Sanguínea X Domínio Social & $-0,355$ & 0,013 \\
s1 Estrutura do Sistema Nervoso & & \\
s110 Cérebro X Domínio Psicológico & $-0,308$ & 0,032 \\
s110 Cérebro X Domínio Ambiental & $-0,366$ & 0,012 \\
\hline
\end{tabular}

Coeficiente de correlação de Sperman; Significância estatística: $\mathrm{p}<0,05$. 
Tabela 2. Fatores Contextuais - Fatores Ambientais X Domínios do WHOQOL - Bref ( $\mathrm{n}=48)$.

\begin{tabular}{|c|c|c|}
\hline Variáveis & Coeficiente (r) & p-valor \\
\hline \multicolumn{3}{|l|}{ d1 Aprendizagem e aplicação do conhecimento } \\
\hline d166 Ler X Domínio Psicológico & $-0,333$ & 0,021 \\
\hline d166 Ler X Domínio Social & $-0,443$ & 0,002 \\
\hline d170 Escrever X Domínio Social & $-0,454$ & 0,002 \\
\hline d172 Calcular X Domínio Físico & $-0,293$ & 0,047 \\
\hline d172 Calcular X Domínio Psicológico & $-0,402$ & 0,005 \\
\hline d172 Calcular X Domínio Social & $-0,458$ & 0,002 \\
\hline d175 Resolver problemas X Domínio Social & $-0,417$ & 0,004 \\
\hline \multicolumn{3}{|l|}{ d2 Tarefas e demandas gerais } \\
\hline d210 Realizar uma única tarefa X Domínio Psicológico & $-0,284$ & 0,049 \\
\hline d210 Realizar uma única tarefa X Domínio Social & $-0,312$ & 0,030 \\
\hline d220 Realizar tarefas múltiplas X Domínio Psicológico & $-0,329$ & 0,022 \\
\hline \multicolumn{3}{|l|}{ d3 Comunicação } \\
\hline d310 Comunicação - recepção de mensagens verbais X Domínio Físico & $-0,345$ & 0,016 \\
\hline d315 Comunicação - recepção de mensagens não verbais X Domínio Social & $-0,311$ & 0,031 \\
\hline d330 Fala X Domínio Social & $-0,384$ & 0,007 \\
\hline d350 Conversação X Domínio Social & $-0,337$ & 0,019 \\
\hline d350 Conversação X Domínio Social & $-0,309$ & 0,032 \\
\hline \multicolumn{3}{|l|}{ d4 Mobilidade } \\
\hline d450 Andar X Domínio Físico & $-0,397$ & 0,006 \\
\hline d450 Andar X Domínio Psicológico & $-0,294$ & 0,042 \\
\hline d450 Andar X Domínio Social & $-0,364$ & 0,010 \\
\hline \multicolumn{3}{|l|}{ d5 Cuidado Pessoal } \\
\hline d510 Lavar-se X Domínio Físico & $-0,305$ & 0,034 \\
\hline d510 Lavar-se X Domínio Ambiental & $-0,358$ & 0,012 \\
\hline d520 Cuidado das partes do corpo X Domínio Físico & $-0,309$ & 0,032 \\
\hline d530 Cuidados relacionados aos processos de excreção X Domínio Físico & $-0,391$ & 0,005 \\
\hline d540 Vestir-se X Domínio Físico & $-0,388$ & 0,006 \\
\hline d540 Vestir-se X Domínio Psicológico & $-0,329$ & 0,022 \\
\hline d540 Vestir-se X Domínio Ambiental & $-0,363$ & 0,010 \\
\hline d550 Comer X Domínio Físico & $-0,449$ & 0,002 \\
\hline d550 Comer X Domínio Psicológico & $-0,361$ & 0,011 \\
\hline d550 Comer X Domínio Ambiental & $-0,341$ & 0,017 \\
\hline d560 Beber X Domínio Físico & $-0,433$ & 0,003 \\
\hline d560 Beber X Domínio Psicológico & $-0,375$ & 0,008 \\
\hline \multicolumn{3}{|l|}{ d6 Vida Doméstica } \\
\hline d570 Cuidar da própria saúde X Domínio Psicológico & $-0,338$ & 0,018 \\
\hline d620 Aquisição de bens e serviços X Domínio Psicológico & $-0,310$ & 0,031 \\
\hline d620 Aquisição de bens e serviços X Domínio Ambiental & $-0,335$ & 0,016 \\
\hline \multicolumn{3}{|l|}{ d7 Relações e Interações Interpessoais } \\
\hline d770 Relações íntimas X Domínio Social & $-0,346$ & 0,015 \\
\hline \multicolumn{3}{|l|}{ d8 Áreas principais da vida } \\
\hline d850 Trabalho remunerado X Domínio Físico & $-0,442$ & 0,002 \\
\hline d850 Trabalho remunerado X Domínio Psicológico & $-0,309$ & 0,032 \\
\hline d850 Trabalho remunerado X Domínio Social & $-0,301$ & 0,037 \\
\hline d850 Trabalho remunerado X Domínio Ambiental & $-0,370$ & 0,009 \\
\hline
\end{tabular}

Coeficiente de correlação de Sperman; Significância estatística: $\mathrm{p}<0,05$. 
Não... depois... não dá mais (escrita).

Bah... nada... nada... a... cabeça fico fraca (cálculo). (S30; em 22/10/2014, no domicílio do sujeito).

As tarefas e as demandas gerais (d2) limitamse a dois domínios - o psicológico e o social. A comunicação (d3), fator importante para as relações humanas, apresentou maior interferência no domínio social (Tabela 2).

As alterações na mobilidade (d4) mostraramse significantes apenas na variável andar (Tabela 2) afetando os domínios físicos $(p<0,006)$, psicológico $(\mathrm{p}<0,042)$ e social $(\mathrm{p}<0,010)$.

O cuidado pessoal (d5) foi afetado em quase todas as suas variáveis, demonstrando as limitações enfrentadas pelos sujeitos após a LEA em atividades básicas do cotidiano, como: tomar banho, alimentar-se e vestir-se. As limitações nas atividades de higiene, como o banho (d510), interferiram nos domínios físicos $(\mathrm{p}<0,034) \mathrm{e}$ ambiental $(\mathrm{p}<0,012)$; o cuidado das partes do corpo (d520) e os relacionados à excreção (d530) comprometeram o domínio físico. As atividades domésticas (d6) influenciaram nos domínios psicológicos e ambientais das pessoas com LEA.

A vida doméstica é um fator importante de ser lembrado; neste estudo houve correlação de duas variáveis: as sequelas causadas no cuidado da própria saúde (que acaba por atingir os domínios psicológicos $[\mathrm{p}<0,018]$ ) e a aquisição de bens e serviços (afetando os domínios psicológi$\cos [\mathrm{p}<0,018]$ e ambiental $[\mathrm{p}<0,016])$.

Nas relações e interações interpessoais (d7), observou-se que as relações mais afetadas pela LEA são as íntimas (d770), causando transtornos no domínio social $(\mathrm{p}<0,015)$. As alterações nas áreas principais da vida ( $\mathrm{d} 8)$ causam grandes transtornos; as atividades de trabalho remunerado (d850) encontraram-se alteradas em todos os domínios do WHOQOL - Bref, ou seja, no físico $(\mathrm{p}<0,002)$, psicológico $(\mathrm{p}<0.032)$, social $(\mathrm{p}<$ $0,037)$ e ambiental $(\mathrm{p}<0,009)-($ Tabela 2$)$. O trabalho (d850), atividade de extrema importância para um sujeito em idade produtiva, apresentou-se com as sequelas mais graves após a LEA, seja pelo afastamento parcial ou total.

Nos domínios físicos $(\mathrm{p}<0,002)$, as sequelas funcionais causaram limitações na mobilidade e na capacidade de trabalho. Os domínios psicológicos $(\mathrm{p}<0,032)$ foram afetados pelas sequelas linguísticos/cognitivas e pelos comprometimentos emocionais. Os rompimentos causados, nos domínios sociais $(\mathrm{p}<0,037)$, estiveram presentes nas relações (familiares, amizades e íntimas). Os domínios de ambiente $(\mathrm{p}<0,009)$ foram afeta- dos pelas sequelas que limitaram o sujeito a atuar sobre o contexto em que estava inserido, não permitindo sua plena interação com todos os aspectos que o circunda (Tabela 2) (confira os relatos de S6 e S36):

Não voltei mais a trabalhar... vontade eu tenho... muito; qualquer função; sim... que não seja muito pesado, eu consigo fazer. (S6; em 31/07/2014, no domicílio do sujeito).

Eu queria voltar a trabalhar, mas a parte que eu trabalho é corte e costura lá na fábrica... eu trabalho cortando. Aí fui na médica... pedi para voltar, mas ela me falou que se eu quisesse voltar ela lavava as mãos dela... porque é perigoso eu voltar agora... Eu sinto muita falta e quero muito voltar a trabalhar... não aguento mais ficar em casa. (S36; em 30/10/2014, no domicílio do sujeito).

No atual momento de vidas dos sujeitos deste estudo, constataram-se perdas em relação aos seus papeis ocupacionais, ainda em um período produtivo de suas vidas; a inatividade em relação ao trabalho foi constatada por afastamentos ou por aposentadorias precoces (por invalidez).

Dos 27 familiares/cuidadores, 77,7\% são mulheres. Os familiares/cuidadores entrevistados apresentam uma média de idade de 52,6 anos, sendo a menor 23 anos e a maior 80 anos. Constatou-se que $96,3 \%$ dos cuidadores eram informais, apenas um era profissional contratado pela família. A distribuição do cuidado constatada, neste estudo, foi de $51,8 \%$ por esposas e $7,4 \%$ por esposos; $11,1 \%$ por filhos; $25,9 \%$ por pais e irmãos e 3,8\% por cuidador formal (Tabela 3).

Foi evidenciado que os familiares/cuidadores sofreram alterações em sua rotina de trabalho após a lesão neurológica de seus familiares $(68,7 \%$ param de trabalhar, $20,2 \%$ continuaram exercendo suas atividades trabalhistas e 11,1\% não trabalhavam) (Tabela 3). Dos cuidadores/ familiares informais, 48,6\% eram empregadas domésticas, 32,9\% tinham outras ocupações, tais como: cabeleireira, auxiliar de fábrica, motorista, técnico de enfermagem, funcionário rural, servente de pedreiro, servidor público, estudante, servente merendeira; $14,8 \%$ eram do lar.

Constatou-se, também, que o nível de escolaridade dos familiares/cuidadores foi relativamente baixo: a maioria tinha Ensino Fundamental Incompleto e Completo $(48,1 \%)$ (Tabela 3 ).

Ao serem questionados sobre alterações na estrutura familiar, 85,2\% dos familiares responderam que houve mudanças após a lesão e o mesmo percentual foi obtido com relação às alterações no cotidiano (Tabela 3). A propósito, na Tabela 4, encontra-se a comparação da QV de sujeitos 
acometidos por LEA e dos familiares/cuidadores participantes, sendo possível afirmar que a interferência da LEA ocorre nos quatro domínios da QV (físico, psicológico, social e ambiental), de ambos os grupos. Os comprometimentos nos domínios físicos dos sujeitos com LEA apresentam uma mediana de 46,4 e a dos familiares/cuidadores de 60,7. Os comprometimentos nos domínios físicos, por sobrecarga física, não foram muito mencionados pelos familiares/cuidadores em seus relatos. As alterações que os familiares/ cuidadores relataram foram nos domínios psicológicos, sociais e ambientais.

Ao analisar os domínios psicológicos, constatou-se interferência em ambos os grupos. Os sujeitos com LEA apresentam mediana 58,3 para este domínio da QV e seus familiares/cuidadores de 70,8 (Tabela 4), assim como no discurso de F14.

Tudo! Tudo! Imagina tudo! A gente era muito triste; a gente não aceitava, agora não, agora a gente sabe conviver com a situação, sabe? Mas foi agora, de um ano para cá que, daí... assim. Tudo mudou, né? bah! Levamos muito tempo para aceitar essa situação, mas hoje eu já aceito, quer dizer...

Tabela 4. Mediana dos domínios de qualidade de vida de sujeitos com Lesão Encefálica Adquirida $(\mathrm{n}=48)$ e seus familiares/cuidadores $(\mathrm{n}=27)$.

\begin{tabular}{lccc}
\hline \multicolumn{4}{c}{ Mediana } \\
\hline & $\begin{array}{c}\text { Sujeitos } \\
\text { com LEA }\end{array}$ & $\begin{array}{l}\text { Familiares/ } \\
\text { Cuidadores }\end{array}$ & p-valor \\
\hline Domínio Físico & 46,4 & 60,7 & $<0,01$ \\
Domínio Psicológico & 58,3 & 70,8 & $<0,01$ \\
Domínio Social & 66,6 & 75,1 & $<0,01$ \\
Domínio Ambiental & 53,1 & 59,3 & $<0,01$ \\
Qualidade de Vida & 54,5 & 66,2 & $<0,01$ \\
\hline
\end{tabular}

Teste Não Paramétrico de Mann-Whitney; Significância estatística: $\mathrm{p}<0,05$.

Tabela 3. Caracterização dos familiares/cuidadores de sujeitos com Lesão Encefálica Adquirida - LEA ( $\mathrm{n}=27)$.

\begin{tabular}{lcc}
\hline \multicolumn{1}{c}{ Variável } & Frequência (n) & Percentual (\%) \\
\hline Familiares/cuidadores & 21 & $77,7 \%$ \\
Mulheres & 6 & $22,3 \%$ \\
Homens & & \\
Tipo de Cuidador & 26 & $96,3 \%$ \\
$\quad$ Formal & 1 & $3,7 \%$ \\
$\quad$ Informal & & \\
Parentesco & 14 & $51,8 \%$ \\
Esposa & 2 & $7,4 \%$ \\
Esposo & 3 & $11,1 \%$ \\
Filhos & 1 & $3,8 \%$ \\
Cuidador Formal & 7 & $25,9 \%$ \\
Pais e irmãos & & \\
Alteração na atividade profissional familiar & & $11,1 \%$ \\
Não trabalhava fora & 4 & $68,7 \%$ \\
Parou de trabalhar após a lesão de seu familiar & 16 & $20,2 \%$ \\
Continuou trabalhando após a lesão de seu familiar & 6 & \\
Escolaridade & & $48,1 \%$ \\
Ensino Fundamental Incompleto & 13 & $25,9 \%$ \\
Ensino Fundamental Completo & 7 & $3,7 \%$ \\
Ensino Médio Incompleto & 1 & $14,8 \%$ \\
Ensino Médio Completo & 4 & $3,7 \%$ \\
Ensino Superior Completo & 1 & $3,7 \%$ \\
Ensino Fundamental Incompleto & 1 & $85,1 \%$ \\
Alteração na estrutura familiar & & $14,8 \%$ \\
Sim & 23 & $85,1 \%$ \\
Não & 4 & $14,8 \%$ \\
Alterações no Cotidiano familiar & 23 & \\
Sim Não & 4 & \\
\hline
\end{tabular}

"Na variável alteração na atividade profissional familiar, desconsiderou-se o cuidador formal. 
aceitar a gente nunca aceita... mas, pelo menos, a gente não chora mais, não sofre mais. (F14; em 24/09/2014, no domicílio do sujeito).

As limitações nos domínios sociais acometeram de forma significativa os sujeitos com LEA $(66,6)$; os familiares/cuidadores $(75,1)$ também apresentam interferências (confira o relato de F9, por exemplo. mostrando o afastamento de algumas atividades que antes eram prazerosas para o casal e a Tabela 4 .

[...] ficou mais dificultoso de sair, a gente podia... né? Sair mais e tudo, né? A gente podia ir visitar mais os conhecidos, os parentes e tudo. Agora a gente faz menos isso. Um bailezinho também agora fica mais difícil, ela não dança mais e coisa. A gente gostava se divertir assim, de sair normal, então... fica mais difícil, né? (F9; em 27/08/2014, no domicílio do sujeito).

As transformações nos domínios ambientais, interferiram na QV de ambos os grupo; na relação deste domínio observou-se que os sujeitos com LEA apresentam uma mediana de 53,1 e para os familiares/cuidadores a interferência foi de 59,3. Ao analisar os relatos dos familiares/ cuidadores, constatou-se que o contexto doméstico sofreu as principais alterações, sendo, muitas vezes, associadas com a alteração das atividades trabalhistas dos familiares/cuidadores (confira os excertos de discursos de F27 e F12:

É Eu tive que deixar de trabalhar para cuidar dele. Agora ele só fica em casa, coisa que antes não acontecia, por que ele trabalhava para fora... ai, ele vinha só aos finais de semana. Então... É isso; agora eu fico mais em função dele! (F27; em 27/10/2014, no domicílio do sujeito).

[...] Eu fazia todo o meu serviço e ainda trabalhava para fora... fazia faxina nas casas, sempre ganhado o meu troquinho e... agora não! Parou tudo [...]. E! Eu tive que deixar de fazer muitas coisas, depois da lesão dele. (F 12; em 11/09/2014, no domicílio do sujeito).

A análise da QV, em seus quatro domínios, revelou que os sujeitos com LEA apresentaram mediana de 54,5 na QV e seus familiares/cuidadores de 66,2. Tais valores revelam que tanto os sujeitos com LEA, quanto os seus familiares/cuidadores, apresentaram índices medianos de QV.

\section{Discussões}

A LEA é uma das principais causadoras de incapacidades em pessoas em idade produtiva ${ }^{3}$, e acomete principalmente sujeitos do sexo masculino ${ }^{13,14}$. Assim como em outros estudos realizados por Falcão et al. ${ }^{15}$, observou-se neste a predominância de sujeitos casados acometidos por LEA, fator que pode atingir a estrutura familiar. Sabese também que a LEA é responsável por $40 \%$ das aposentadorias precoces no Brasil ${ }^{16}$, sendo assim, a lesão neurológica gera consequências negativas no sujeito e seus familiares ${ }^{3}$.

O nível escolar dos sujeitos com LEA deste estudo pode ser considerado baixo (Ensino Fundamental Incompleto); um fator agravante associado a hábitos precários de saúde (alimentação pobre em nutrientes, negligência de práticas de atividades físicas, consumo de drogas lícitas [álcool e tabaco], por exemplo) ${ }^{17}$.

Sujeitos acometidos por LEA podem apresentar dificuldades cognitivas ${ }^{18}$, as quais foram apresentadas na Tabela 1 e no relato do S13. Distúrbios nas Funções Mentais interferiram nos quatro domínios da QV, causando impactos significativos nas relações familiares e/ou sociais, nas AVD, e ainda, foram responsáveis por um grande número de afastamentos das atividades laborais, tal como estudo realizado por Oliveira e Silveira ${ }^{19}$.

As Funções do Coração e da Pressão Sanguínea foram referidas pelos sujeitos deste estudo como limitadoras dos domínios físicos e sociais, visto que enfrentam o impacto da descoberta de um comprometimento crônico, o rótulo da patologia e suas consequências negativas sobre o bem estar, além da medicalização contínua. A perda do bem estar social repercute expressivamente no estilo de vida desses sujeitos; as patologias do coração e da pressão sanguínea causam diversas transformações na vida do sujeito, modificando sua relação consigo mesma e com o mundo, exigindo reconstrução de sua identidade ${ }^{18}$.

As complicações relacionadas à Pressão Sanguínea atingem os aspectos psicológico, familiar, social e/ou econômico, devido à probabilidade de complicações no longo prazo. Deste modo, reforçam-se as discussões que defendem uma análise ampliada do sujeito, em que a patologia e suas complicações orgânicas não devem ser analisadas de forma isolada das demais dimensões da vida ${ }^{20}$.

O cérebro é a principal Estrutura Corporal afetada pela LEA (conforme explicitado na Tabela 1), assim, suas disfunções podem comprometer as capacidades funcionais em todas as esferas da vida ${ }^{21}$, por exemplo: prejuízos nas atividades de leitura, escrita, cálculo e resolução de problemas, e nos processos de comunicação oral ${ }^{19}$. Os comprometimentos cerebrais e as limitações causadas na qualidade de vida envolvem: orientação espaço-temporal, memória, atenção, linguagem 
e funções executivas, havendo intrínseca relação com o desempenho nas atividades cotidianas ${ }^{22}$.

As restrições na mobilidade (andar) afetam os domínios físico, psicológico e social do sujeito com LEA, pois não limitam somente o ato de ir e vir, mas se estendem às atividades de cuidado pessoal, de vida doméstica e de trabalho. Para Rangel et al. ${ }^{23}$, a LEA pode prejudicar as atividades mais simples e íntimas (como as atividades de higiene), até as mais complexas (as de trabalho).

As atividades profissionais são interrompidas no período imediato à LEA, sendo as incapacidades motoras e linguístico/cognitivas as mais comuns e visíveis ${ }^{24}$. O impacto psicológico gerado pelo prejuízo funcional pode disparar respostas emocionais depressivas, as quais, numa dinâmica de retroalimentação, podem dificultar aquisições funcionais ${ }^{25}$.

Com intuito de evitar prejuízos linguístico/ cognitivas, funcionais e agravantes psico/emocionais, causadas pelo afastamento da atividade laboral, Teixeira e Guimarães ${ }^{26}$ defendem o retorno ao trabalho; explicam que tal condição é uma oportunidade que o sujeito com LEA tem de reconstruir sua vida após a lesão. O sujeito com LEA pode retornar à mesma função que exercia antes da lesão (caso esta não exponha a riscos de acidentes trabalhistas) ou mudar de função/ profissão, a partir de cursos de qualificação profissional, exercendo assim atividades que não o exponham a riscos.

O processo de reinserção laboral, no Brasil, é dificultado pelas barreiras previdenciárias e pelo desconhecimento dos direitos garantidos pela legislação brasileira ${ }^{26}$. Sabe-se dos direitos que garantem a reabilitação, (re)educação e (re) adaptação profissional e social e reinserção no mercado de trabalho ${ }^{27}$, porém os profissionais da reabilitação não os incentivam e os sujeitos com LEA não os desfrutam (vide o caso da maioria dos sujeitos deste estudo).

Do nosso ponto de vista, além das barreiras citadas anteriormente, os sujeitos com LEA, quando reinserido no trabalho, têm de enfrentar o despreparo das empresas, muitas vezes relacionadas a barreiras físicas (arquitetônicas, ergonômicas) e atitudinais (discriminação, estigmas, estereótipos e preconceitos). Sabe-se também que a nova condição de vida do sujeito com LEA exige transformações importantes e significativas no ambiente domiciliar, entre elas aparece a figura do familiar/cuidador.

Este estudo revelou a predominância de cuidadores/familiares do sexo feminino (77,7\%), em concordância com outros estudos apresen- tados por Lemos et al. ${ }^{28}$, Araújo et al. ${ }^{29}$, Pinto e Nations ${ }^{30}$ e Martins et al. ${ }^{31}$. O percentual é muito próximo do estudo de Araújo et al. ${ }^{29}$, que apontou $80 \%$ de mulheres cuidadoras e $20 \%$ de homens. Evidenciou-se que o ato de cuidar é fundamentalmente desempenhado por familiares, sendo a esposa a principal cuidadora. Ao lado de outro estudo $^{19}$, este aponta para o papel de cuidador desempenhado pela esposa, apesar de todas as alterações estruturais ocorridas na família nas últimas décadas, pois as mulheres da família continuam a ser as principais responsáveis pelo cuidado informal dos sujeitos que apresentam algum tipo de incapacidade ${ }^{31}$.

Conforme o estudo de Araújo et al. ${ }^{29}$, tal condição, limita a vida social dos cuidadores/familiares, reduzindo os encontros com parentes e amigos.

Os familiares/cuidadores deste estudo relataram alterações no cotidiano e na estrutura familiar, assim como constatado no estudo de Pinto e Nations ${ }^{30}$. Tais autores evidenciaram um percentual ligeiramente mais alto $(92,6 \%)$ que o deste estudo. Concluem que os familiares/ cuidadores estão expostos a mudanças no estilo de vida, resultantes do trabalho extra e da perda de independência, por exemplo, o que pode acarretar depressão, frustração, sentimentos de inutilidade e de solidão. A sobrecarga do familiar/cuidador pode gerar complicações físicas, mentais, emocionais e financeiras ${ }^{32}$. A estrutura familiar pode sofrer alterações como inversões de papeis, gerando situações estressoras, desgastantes e frustrantes ${ }^{21}$.

O nível de escolaridade dos familiares/cuidadores deste estudo foi baixo e, segundo Cavalcan$\mathrm{te}^{33}$, a escolaridade influencia os sentimentos dos familiares/cuidadores - a pouca aprendizagem escolar dificulta a compreensão do que acontece com o sujeito lesionado neurológico. Concordamos com o referido autor que afirma que os sentimentos dos cuidadores são consequências de uma interação complexa de fatores como convivência, medo e empatia, associado à condição de absorver informações sobre a LEA e suas consequências.

Convém considerar que o desempenho do “papel de cuidador" é, na maioria das vezes, realizado sem o preparo adequado, fato que pode gerar conflitos pessoais, familiares e até mesmo no trabalho, caso os responsáveis/cuidadores desempenhem outras funções no dia-a-dia ${ }^{31}$. Tais autores encontraram que $48,2 \%$ dos participantes, de seu estudo, dividem o seu tempo entre o trabalho e o cuidado e que $51,8 \%$ pararam de 
trabalhar após a lesão de seu familiar. Geralmente, o familiar/cuidador tem problemas com o emprego, abandonando-o ou reduzindo a jornada de trabalho, o que acaba repercutindo na situação financeira familiar ${ }^{31,29}$ e na qualidade de vida do familiar/cuidador.

Os familiares/cuidadores deste estudo não citaram muitas alterações em seus domínios físicos, mas, no estudo realizado por Gonçalves et al. ${ }^{34}$, os cuidadores reconheceram a tarefa como estressante, dado ao excesso de atenção dedicado ao sujeito com LEA, o que implica pouco tempo para cuidar de si mesmos e menos tempo de descanso.

A sobrecarga emocional, também mencionada neste estudo, interfere na qualidade de vida do familiar/cuidador; é ameaçada pelo estresse gerado pela responsabilidade de cuidar ${ }^{35}$. Outro fator que pode gerar sobrecarga emocional é o afastamento do sujeito com LEA do trabalho, fator que não é visto pelos familiares/cuidadores como temporária para tratar de uma doença adquirida; é vista, na maioria das vezes, como definitiva, situação carregada de valores e sentimentos negativos $^{36}$.

Estudo realizado por Di Giulio e $\mathrm{Chun}^{37}$ aponta que as afasias, sequelas da LEA, geram estresse aos familiares/cuidadores pela dificuldade de comunicação; discutem a influência no cotidiano de cuidadores informais (cônjuges, filhos, pais, irmãos, parentes mais próximos) e cuidadores formais (técnicos de enfermagem, enfermeiros), destacando as consequências das sequelas na atividade de cuidado.

Outros fatores da qualidade de vida comprometidos após uma LEA são os domínios ambientais, principalmente o doméstico. O domicilio do sujeito com LEA, muitas vezes, necessita de adaptações que proporcionem autonomia; estas podem fornecer conforto e segurança para os sujeitos com LEA e mais liberdade para os familiares/ cuidadores ${ }^{38}$. Porém, podem ser um agravante na situação econômica familiar, pois algumas mudanças são dispendiosas; somado a isso tem-se o afastamento das atividades laborais, a dedicação aos processos de reabilitação, gerando-se assim diminuição da renda doméstica ${ }^{39}$.

Note-se que este estudo confirmou um conjunto de efeitos causado pela LEA na vida do sujeito por ela acometido e na sua dinâmica familiar. Os achados remetem a novas demandas de ambos os grupos - sujeitos e famílias - bem como impelem os profissionais a novos paradigmas de como lidar com a realidade de sujeitos com LEA e seus familiares.

\section{Conclusão}

Pôde-se concluir que o surgimento de uma LEA, de forma súbita, causa sequelas incapacitantes: desorganiza o sujeito e sua família, afetando a sua qualidade de vida em todos os aspectos, assim, exigindo que seja adotada uma ação imediata de enfrentamento.

Este estudo evidenciou que a CIF e o WHO$Q O L$ - BREF são ferramentas importantes para analisar as condições de saúde e qualidade de vida de sujeitos acometidos por LEA, bem como a de seus familiares. Constatou-se a íntima relação entre os aspectos da Funcionalidade e Incapacidade (Funções e Estruturas do Corpo) dos Fatores Contextuais - Fatores Ambientais com os Domínios do WHOQOL - BREF.

Nas funções do corpo observou-se que as alterações nas Funções Mentais refletem significativamente nos domínios da qualidade de vida. As Funções do Sistema Cardiovascular, Hematológico, Imunológico e Respiratório, mais especificadamente as do coração e a pressão sanguínea demonstraram interferências na vida do sujeito. A principal Estrutura Corporal lesada que atinge os domínios da qualidade de vida é o cérebro. Nos Fatores Contextuais-Fatores Ambientais, as áreas de aplicação do conhecimento, tarefas e demandas gerais, comunicação, mobilidade, cuidado pessoal, vida doméstica, relação e Interações Interpessoais e as áreas principais da vida, foram as mais comprometidas nos quatro domínios de qualidade de vida no sujeito pós LEA. Assim, pode-se notar as intensas alterações que a LEA trás para a vida profissional do sujeito acometido, o qual, após a lesão, passa processos de reabilitação e de ressignificação pessoal e profissional, buscando a identidade abalada, ou seja, procurando encontrar novos sentidos diante do novo contexto que se apresenta.

Este estudo chamou a atenção para as condições de vida dos familiares/cuidadores que, também, são "atropelados" por uma nova realidade. Destacou a conveniência de os profissionais se ocuparem de uma atenção integral a estes usuários e suas famílias, por meio da observação das necessidades reais destes sujeitos, visando à diminuição do impacto da LEA para uma melhor qualidade de vida e para o retorno dos mesmos às atividades de trabalho.

Devido à importância do tema, acredita-se que estudos posteriores possam aprofundar conhecimentos relativos aos tratamentos de reabilitação. 


\section{Colaboradores}

AS Ponte é a autora do estudo e participou da concepção do texto manuscrito, organização de fontes e/ou análises e redação do texto. E Fedosse é orientadora do estudo, e participou da concepção do texto manuscrito e revisão.

\section{Agradecimentos}

À Coordenação de Aperfeiçoamento de Pessoal de Nível Superior (CAPES) pelo financiamento.

\section{Referências}

1. Abaquim MLM, Lima MP, Ciasca SM. Avaliação neuropsicológica de sujeitos com lesão cerebral: uma revisão bibliográfica. Rev. psicopedag 2012; 28(89):236-243.

2. Organização Mundial da Saúde (OMS). Classificação Internacional de Funcionalidade, Incapacidade e Saúde - CIF. São Paulo: Editora da Universidade de São Paulo; 2008.

3. Smith J, Borchelt M, Maier H, Jopp D. Health and well-being in the young and oldest old. Journal of Social Issues 2002; 58(4):715-732.

4. Whoqol Group. Development of the WHOQOL: Rationale and current status. International Journal of Mental Health 1994; 23(3):24-56.

5. Carod-Artal FJ. Determining quality of life in stroke survivors. Expert Rev Pharmacoecon Outcomes Res 2012; 12(2):199-211.

6. Bahia MM, Chun RYS. Qualidade de vida na afasia: diferenças entre afásicos fluentes e não fluentes usuários de Comunicação Suplementar e/ou Alternativa. Audiol Commun Res 2014; 19(4):352-359.

7. Fortes ACG, Neri AL. Eventos de vida e envelhecimento humano. In: Néri AL, Yassuda MS, Cachioni M, organizadores. Velhice bem sucedida: aspectos afetivos e cognitivos. Campinas: Papirus; 2004. p. 51-70.

8. Panhoca I, Gonçalves CAB. Afasia e qualidade de vida - consequências de um acidente vascular cerebral na perspectiva da fonoaudiologia. Arq. Ciênc. Saúde 2009; 13(2):147-153.

9. Brasil. Decreto-Lei n. ${ }^{\circ}$ 5.452, de $1^{\circ}$ de maio de 1943. Aprova a Consolidação das Leis do Trabalho. Diário Oficial da União 1943; 01 mai.

10. Di Nubila HBV, Buchalla CM. O papel das Classificações da OMS - CID e CIF nas definições de deficiência e incapacidade. Rev. Bras. Epidemiol 2008; 11(2):324335.

11. Castro MMLD, Hökerberg YHM, Passos SRL. Validade dimensional do instrumento de qualidade de vida WHOQOL-BREF aplicado a trabalhadores de saúde. Cad Saude Publica 2013; 29(7):1357-1369.

12. Bardin L. Análise de conteúdo. Lisboa: Edições 70; 2009.

13. Schuster RC, Polese JC, Silva SLA, Perin V, Seben YP. Caracterização de internações hospitalares por acidente vascular encefálico na cidade de Passo Fundo - RS. Con Scientia e Saúde 2009; 8(4):581-585.

14. Gaudêncio TG, Leão GM. A Epidemiologia do Traumatismo Crânio Encefálico: Um Levantamento Bibliográfico no Brasil. Rev Neurocienc 2013; 21(3):427-434.

15. Falcão IV, Carvalho EMF, Lessa FJ D, Leite VMM. Acidente Vascular Cerebral Precoce: Implicação para o adulto em idade produtiva Atendidos no sistema Único de Saúde. Rev Bras Matern Infantil 2004; 4(1):95-102.

16. Abramczuk B, Villela, E. A luta contra o AVC no Brasil. Com Ciência [periódico na Internet]. 2009 [acessado 2015 maio 25]; 109: [cerca de 4p.] Disponível em: http://www.comciencia.br/comciencia/handler.php?section $=8$ \&edicao $=47 \&$ id $=582 \&$ tipo $=0$

17. Martin RSS, Godoy I, Franco RJS, Martin LC, Martins AS. Influência do nível socioeconômico sobre os fatores de risco cardiovascular. JBM 2014; 102(2):34-37.

18. Fava SMCL, Zago MMF, Nogueira MS, Dázio EMR. Experiência da doença e do tratamento para a pessoa com hipertensão arterial sistêmica: um estudo etnográfico. Rev. Latino-Am. Enfermagem 2013; 21(5):1-7. 
19. Oliveira AIC, Silveira KRM. Utilização da CIF em pacientes com sequelas de AVC. Rev Neurocienc 2011; 19(4):653-662.

20. Langdon EJ, Wiik FB. Antropologia, saúde e doença: uma introdução ao conceito de cultura aplicado às ciências da saúde. Rev. Latino-Am. Enfermagem 2010; 18(3):1-9.

21. Farias HHQ, Vall J, Oliveira DC, Braga VAB. O ser cuidador da pessoa idosa com lesão cerebral: um estudo teórico. Rev. RENE 2005; 6(3):112-119.

22. Ferro AO, Lins AES, Trindade Filho EM. Comprometimento cognitivo e funcional em pacientes acometidos de acidente vascular encefálico: Importância da avaliação cognitiva para intervenção na Terapia Ocupacional. Cad. Ter. Ocup. UFSCar 2013; 21(3):521-527.

23. Rangel ESS, Belasco AGS, Diccini S. Qualidade de vida de pacientes com acidente vascular cerebral em reabilitação. Acta paul. Enferm 2013; 26(2):205-212.

24. Adams RJ, Mckie VC, Hsu L, Files B, Vichinsky E, Pegelow C. Prevention of a first stroke by transfusions in children with sickle cell anemia and abnormal results on transcranial Doppler ultrasonography. $N$ Engl J Med. 1998; 11(5):5-11.

25. Terroni LMN, Leite CC, Tinone G, Fráguas Junior R. Depressão pós-avc: fatores de risco e terapêutica terapêutica antidepressiv antidepressiva. Rev Assoc Med Bras 2003; 49(4):450-459.

26. Teixeira AM, Guimarães L. Vida revirada: deficiência adquirida na fase adulta produtiva. Rev. Mal-Estar Subj 2006; 6(1):182-200.

27. Brasil. Lei no 8.213, de 24 de julho de 1991. Dispõe sobre os Planos de Benefícios da Previdência Social e dá outras providências. Diário Oficial da União. 1991; 24 jul.

28. Lemos ND, Gazzola JM, Ramos LR. Cuidando do paciente com Alzheimer: o impacto da doença no cuidador. Saúde Soc 2006; 15(3):170-179.

29. Araújo JS, Silva SED, Santana ME, Conceição VM, Vasconcelos EV. O perfil representacional dos cuidadores de pacientes acometidos por Acidente Vascular Cerebral. Revista Eletrônica Gestão \& Saúde. 2012; 3(3):852864.

30. Pinto JMS, Nations MK. Cuidado e doença crônica: visão do cuidador familiar no Nordeste brasileiro. Cien Saude Colet 2012; 17(2):521-528.
31. Martins T, Ribeiro JP, Garret C. Estudo de validação do questionário de avaliação da sobrecarga para cuidadores informais. Psicol Saude Doencas 2003; 4(1):131-148.

32. Costa MLG, Fedosse E, Lefèvre AP. Doenças Crônicas Não Transmissíveis - Cuidado em Fonoaudiologia. In: Marchesan IQ, Silva HJ, Tomé MC, organizadores. Tratado das Especialidades em Fonoaudiologia. São Paulo: Guanabara Koogan; 2014. p. 806-813.

33. Cavalcante LF. Gênero, família e trabalho no Brasil. Rio de Janeiro: FGV; 2010.

34. Gonçalves LHT, Costa MAM, Martins MM, Nassar SM, Zunin R. The family dynamics of elder elderly in the context of Porto, Portugal. Rev Latino-Am Enfermagem 2011; 19(3):458-466.

35. Araújo JS, Silva SED, Conceição VM, Santana ME, Vasconcelos EV. A obrigação de (des)cuidar: representações sociais sobre o cuidado à sequelados de acidente vascular cerebral por seus cuidadores. Revista Mineira de Enfermagem 2011; 16(1):98-105.

36. Brasil. Ministério da Saúde (MS). Lesões por esforços repetitivos (LER): Distúrbios osteomusculares relacionados ao trabalho (DORT). Brasília: MS; 2001.

37. Di Giulio RM, Chun RYS. Impacto da afasia na perspectiva do cuidador. Distúrb Comun 2014; 26(3):541549.

38. Gindri G, Argimon IIL, Fonseca RP. O cuidado após um acidente vascular cerebral: reflexões do indivíduo à saúde pública. Revista Grifos 2010; 19(28):89-105.

39. Silveira TM, Caldas CP, Carneiro TF. Cuidando de idosos altamente dependentes na comunidade: um estudo sobre cuidadores familiares principias. Cad Saude Publica 2006; 22(8):1629-16380.

Artigo apresentado em 10/03/2016

Aprovado em 12/07/2016

Versão final apresentada em 14/07/2016 